\title{
Infant coronary artery bypass grafting completely under surgical microscope
}

\author{
Yusuke Iwata, MD, ${ }^{\mathrm{a}}$ Takamasa Takeuchi, MD, ${ }^{\mathrm{b}}$ Takeshi Konuma, MD, ${ }^{\mathrm{b}}$ Kikuko Obase, $\mathrm{MD},{ }^{\mathrm{c}}$ and \\ Kiyoyuki Eishi, MD, ${ }^{\mathrm{c}}$ Gifu, Nagano, and Nagasaki, Japan
}

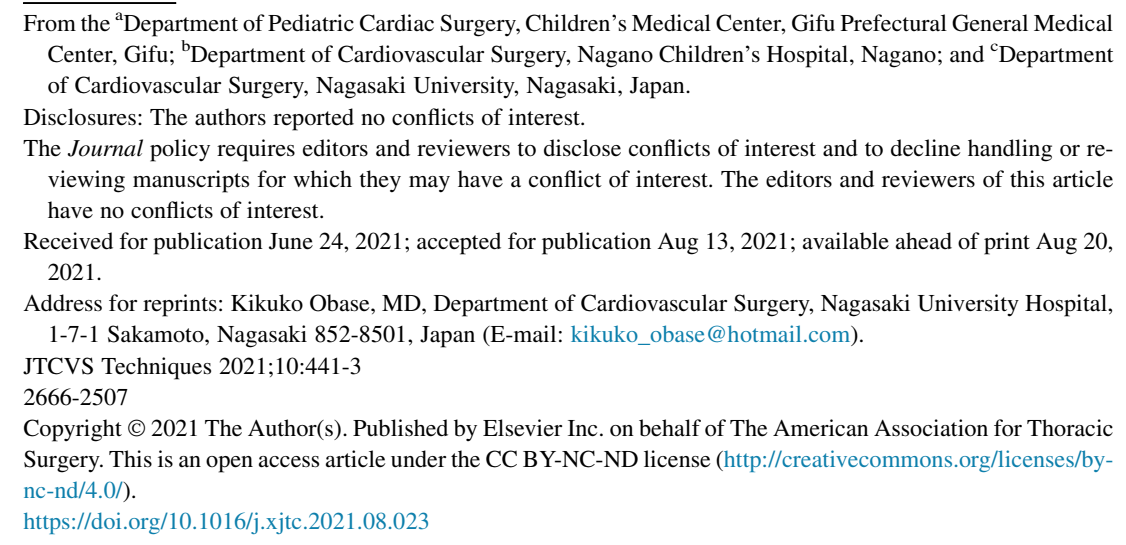

Video clip is available online.

With an increase in congenital heart surgeries such as arterial switch operation (ASO) or the Ross procedure, coronary artery bypass grafting $(\mathrm{CABG})$ in infants has become an increasingly important option because of coronary obstruction as a result of coronary manipulation. ${ }^{1,2}$ CABG in infants includes technical challenges associated with operating on small vessels. A microscope is, therefore, a promising tool to assist CABG in infants in achieving successful coronary revascularization. ${ }^{3,4}$ This case series introduces 4 infants who underwent total microscopic CABG. The ages were 3 months in 2 cases, 4 months in 1 , and 9 months in 1, weighing 3.2 to $4.8 \mathrm{~kg}$ (average, $4.0 \mathrm{~kg}$ ). Informed written consents were provided from the parents of each case.

\section{SURGERY}

For harvesting the internal mammary artery, magnifying glasses were used in cases 1,2, and 3, and microscope was used in case 4 . The anastomosis was performed with the infant under cardiac arrest, placing single interrupted sutures with 10-0 or 9-0 PROLENE (Ethicon, Somerville, NJ). The toe and heel of the graft were sutured with 9-0 PROLENE. In total, 12 to 16 sutures were placed. The surgical microscope enabled up to $20 \times$ auto focus magnification and microscopic CABG.

\section{CASE REPORTS}

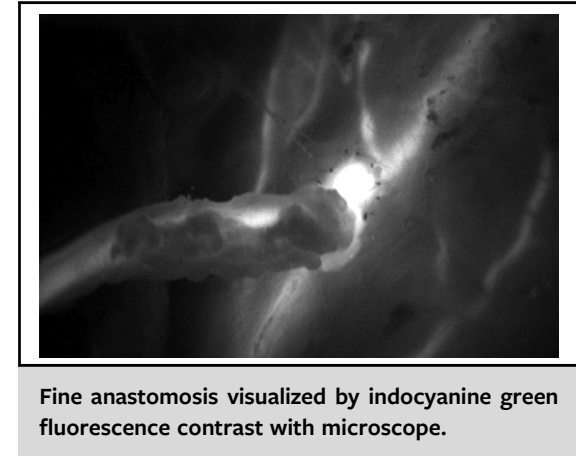

\begin{abstract}
CENTRAL MESSAGE
Coronary artery bypass grafting $(\mathrm{CABG})$ in infants includes technical challenge, but microscopic surgery with sufficient experience leads to successful CABG, with avoiding technical errors.
\end{abstract}

See Commentaries on pages 444 and 446.

hands-free manipulations such as magnification or minute movement of the visual field. Surgery was performed by a surgeon (K.E.) with more than 3 decades of experience in

After the spontaneous beating resumed, good anastomosis was confirmed in all 4 cases using the indocyanine green fluorescence contrast function attached to the microscope (Figure 1). In all cases, postoperative angiography revealed excellent patencies of all bypasses.

The average vessel diameters of 4 cases at near the anastomosis measured by the pre- or postoperative coronary angiography were $1.1 \mathrm{~mm}$ (ranged 0.6-1.3) in coronary artery and $1.1 \mathrm{~mm}$ (ranged $0.8-1.3$ ) in internal thoracic artery.

Case 1 was a previously healthy female infant. She suddenly presented dyspnea at the age of 6 months and developed heart failure requiring mechanical ventilator support. Echocardiogram showed enlarged left ventricular diastolic dimension of $38 \mathrm{~mm}$ and left ventricular ejection fraction (LVEF) of $40 \%$. LVEF reduced to $21 \%$ in a couple of months. Her hemodynamic function was catecholamine 

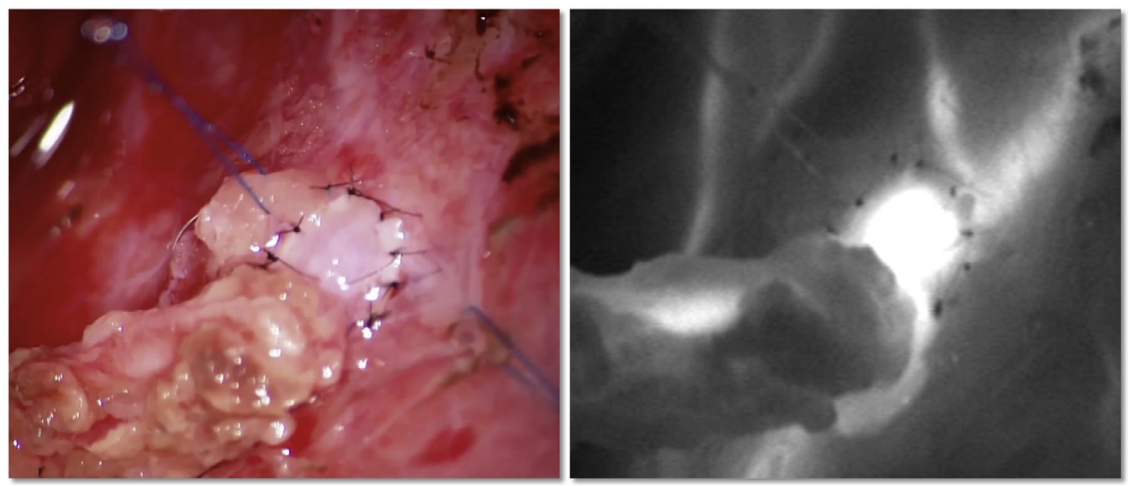

FIGURE 1. Completed anastomosis in case 2 (left) and corresponding indocyanine green fluorescence contrast image (right).

dependent and had difficulty in weaning from the ventilator. Coronary angiography at 9 months revealed $99 \%$ stenosis of hypoplastic left main trunk (LMT). Then, left internal thoracic artery (LITA)-to-left anterior descending artery (LAD) bypass was performed. LVEF recovered to $46 \%$, and she was weaned from ventilator on postoperative day (POD) 14 and discharged on POD 234.

Case 2 was a boy diagnosed with transposition of the great arteries (TGA) who underwent balloon atrial septostomy immediately after birth. ASO was performed at 15 days, and he was discharged on POD 21 in good condition. He developed acute heart failure with reduced LVEF of $23 \%$ on POD 42 . Urgent coronary angiography revealed $90 \%$ stenosis at the ostium of LMT. Then, a LITA-toLAD bypass was performed at 4 months (Video 1). LVEF improved to $53 \%$, and he was weaned from ventilator on POD 5 and discharged on POD 83.

Case 3 was a boy diagnosed as having TGA immediately after birth. ASO was performed at 18 days, and he was discharged on POD 27. Acute heart failure was developed on
POD 64 and coronary angiography depicted $90 \%$ stenosis of proximal LMT. At 28 days after readmission, he went into cardiogenic shock and required extracorporeal membrane oxygenation support. LITA-LAD bypass was performed the following day. $\mathrm{He}$ was taken off extracorporeal membrane oxygenation during surgery and was weaned from the ventilator at POD 8. LVEF at discharge (POD 43) was 70\%.

Case 4 underwent balloon atrial septostomy for TGA at the age of 5 days. ASO with ventricular septal defect closure was performed at 11 days. He was weaned from the mechanical ventilator at 39 days under catecholamine support. Coronary angiography at 2 months showed hypoplastic but patent left coronary artery. The left ventricle was fed by right coronary artery, which had $90 \%$ stenosis at its ostium. Right internal thoracic artery-to-right coronary artery bypass was performed at the age of 3 months (Video 2). He was weaned from ventilator the following day of the surgery and discharged from hospital on POD 81.

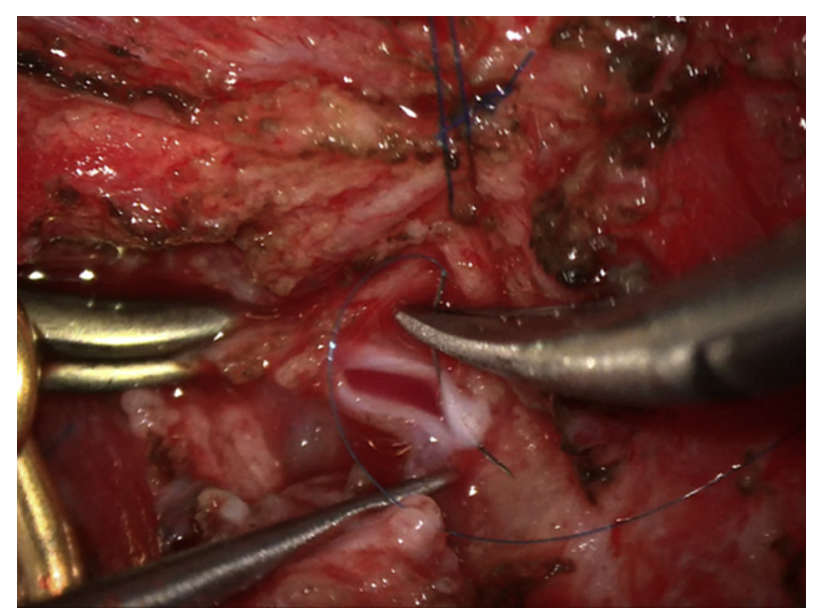

VIDEO 2. Intraoperative video in case 4. Video available at: https://www jtcvs.org/article/S2666-2507(21)00580-0/fulltext. 


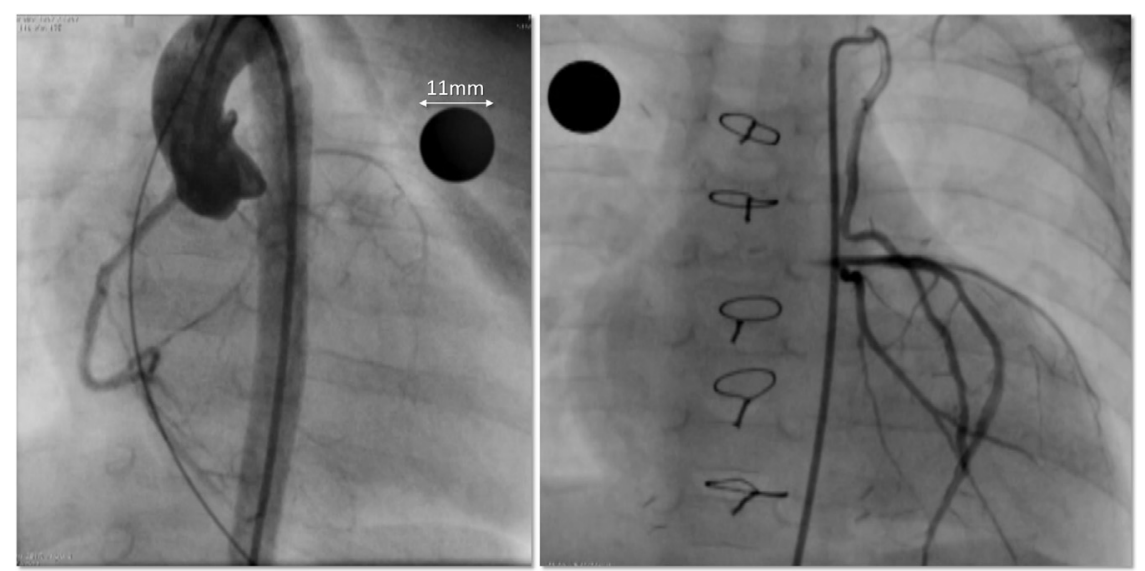

FIGURE 2. Preoperative (left) and postoperative (right) coronary angiography in case 1.

Cases 2, 3, and 4 are now 8 years, 3 years, and 8 months old, respectively, and are growing and developing normally. Despite the excellent patency of the bypass confirmed by the angiogram (Figure 2), however, case 1 suddenly died 2 months after discharge.

This case series was not required to be approved by the institutional review board of the Nagasaki University because the cases was not involved in analytical activity. Informed written consents were provided from the parents of each case.

\section{COMMENT}

Successful coronary revascularization and improvement of cardiac function were achieved in all 4 cases with totally microscopic assisted CABG. Because of technical concerns, some institutions perform microscopic surgery in collaboration with plastic surgeons, ${ }^{3}$ but as our result shows, with sufficient experience, microscopy-assisted infant $\mathrm{CABG}$ was feasible with avoidance of technical errors. ${ }^{4}$ Short term, our results are satisfactory, and further follow-up is needed. We anticipate that our preferred single interrupted sutures would lead to the growth of the anastomosis orifice along to the somatic growth of the children.

\section{References}

1. Mavroudis C, Backer CL, Muster AJ, Pahl E, Sanders JH, Zales VR, et al. Expanding indications for pediatric coronary artery bypass. J Thorac Cardiovasc Surg. 1996;111:181-9.

2. Kitamura S. Pediatric coronary artery bypass surgery for congenital heart disease. Ann Thorac Surg. 2018;106:1570-7.

3. Catapano J, Zuker R, Honjo O, Borschel G. Microvascular coronary artery repair and grafting in infancy and early childhood. Oper Tech Thorac Cardiovasc Surg. 2015;20:148-61.

4. Kitamura $S$. A new arena in cardiac surgery: pediatric coronary artery bypass surgery. Proc Jpn Acad Ser B Phys Biol Sci. 2018;94:1-19. 